\title{
SARS-CoV-2-associated Guillain-Barré syndrome in four patients: what do we know about pathophysiology?
}

\author{
Antoine Guilmot $^{1,5} \cdot$ Sofia Maldonado Slootjes ${ }^{1,2} \cdot$ Véronique Bissay $^{2} \cdot$ Nicolas Dubuisson $^{1,3} \cdot$ Clémence de Broglie $^{4}$. \\ Michel Gille ${ }^{5}$
}

Received: 20 April 2021 / Accepted: 19 August 2021 / Published online: 2 September 2021

(c) Belgian Neurological Society 2021

\begin{abstract}
Background A growing number of Guillain-Barré syndrome (GBS) and Miller Fisher Syndrome (MFS) cases following severe acute respiratory syndrome coronavirus 2 (SARS-CoV-2) infection are reported. Nevertheless, this association is still debated, and pathophysiology remains unclear.

Methods Between April and December 2020, in three hospitals located in Brussels, Belgium, we examined four patients with GBS following SARS-CoV-2 infection.

Results Neurological onset occurred 3 weeks after SARS-CoV-2 symptoms in all patients. Three patients presented with acute inflammatory demyelinating polyneuropathy (AIDP) and had negative anti-ganglioside testing: two suffered from a severe SARS-CoV-2 infection and had good clinical outcome after intravenous immunoglobulin (IVIG) treatment; one with mild SARS-CoV-2 infection had spontaneously favorable evolution without treatment. The fourth patient had critical SARS-CoV-2 infection and presented acute motor and sensory axonal neuropathy (AMSAN) with clinical features highly suggestive of brainstem involvement, as well as positive anti-ganglioside antibodies (anti-GD1b IgG) and had partial improvement after IVIG.

Conclusions We report four cases of SARS-CoV-2-associated GBS. The interval of 3 weeks between SARS-CoV-2 symptoms and neurological onset, the clinical improvement after IVIG administration, and the presence of positive anti-ganglioside antibodies in one patient further support the hypothesis of an immune-mediated post-infectious process. Systematic extensive antibody testing might help for a better understanding of physiopathology.
\end{abstract}

Keywords Guillain-Barré syndrome · SARS-CoV-2 · Case series · Anti-gangliosides · Anti-GD1b · Pathophysiology

Michel Gille

m.gille@cdle.be

1 Department of Neurology, Cliniques Universitaires SaintLuc, Université Catholique de Louvain, Avenue Hippocrate 10, 1200 Brussels, Belgium

2 Department of Neurology, Universitair Ziekenhuis Brussel (UZ Brussel), Vrije Universiteit Brussel (VUB), Laarbeeklaan 101, 1090 Brussels, Belgium

3 Neuromuscular Reference Center, Cliniques Universitaires Saint-Luc, Avenue Hippocrate 10, 1200 Brussels, Belgium

4 Department of Physical Medicine and Rehabilitation, Cliniques de l'Europe, Avenue de Fré 206, 1180 Brussels, Belgium

5 Department of Neurology, Cliniques de l'Europe, Avenue de Fré 206, 1180 Brussels, Belgium

\section{Introduction}

Since the beginning of the COVID-19 outbreak, there has been a growing number of reports of Guillain-Barré syndrome (GBS) and Miller Fisher Syndrome (MFS) following severe acute respiratory syndrome coronavirus 2 (SARS$\mathrm{CoV}-2$ ) infection, suggesting a post-infectious immunemediated process [1]. However, the association between SARS-CoV-2 and GBS is still debated since a recent epidemiological study found no association between both entities [2]. In addition, pathophysiology remains unclear as there is no clear evidence of structural homology between SARS-CoV-2 and nerve compounds to support a molecular mimicry mechanism. Moreover, anti-ganglioside antibodies, which play a central role in pathogenesis-at least in the axonal forms-of GBS, have only been reported in a few cases [3]. Although there is no direct homology between 
SARS-CoV-2 structure proteins and any axonal or myelin surface proteins, it has been suggested that SARS-CoV-2 binds to respiratory tract gangliosides through its spike protein. Cross-reactivity between epitopes on SARS-CoV-2 spike-bound gangliosides and surface peripheral nerve glycolipids is currently considered as a pathophysiological hypothesis [4].

\section{Methods}

Between April and December 2020, four patients with a diagnosis of GBS according to the Brighton criteria, occurring after SARS-CoV-2 infection, were examined at Cliniques universitaires Saint-Luc, Universitair Ziekenhuis Brussel and Cliniques de l'Europe-Saint-Michel, three hospitals located in Brussels, Belgium. A positive diagnosis of COVID-19 infection was established by SARS-CoV-2 PCR assay of nasopharyngeal swab. Anti-ganglioside antibodies were tested by enzyme-linked immunosorbent assay (ELISA) for IgG and IgM antibodies against single gangliosides GM1, GM2, GM3, GM4, GD1a, GD1b, GD2, GD3, GT1a, GT1b, GQ1b and anti-sulfatides. Clinical and ancillary test descriptions were retrieved by the authors, who examined the patients. Consent was obtained from each patient for publication.

\section{Results}

Clinical characteristics and laboratory findings of the four patients with SARS-CoV-2-associated GBS are detailed in Table 1. The electrodiagnosis (EDX) findings are detailed in the Supplementary Appendix (S1-S4).

All patients presented with sensorimotor symptoms and tendon areflexia about 3 weeks (20-24 days) after documented COVID-19 infection. SARS-CoV-2 infection was associated with a severe pulmonary disease in three of them. No other GBS triggering event was identified in all patients. CSF examinations revealed albuminocytologic dissociation in two patients (case 1 and case 2 ) and an increased albumin quotient in one (case4). Positive serum anti-GD1b antibodies were found in one patient (case4). The latter was the only patient showing brainstem involvement, with EDX (S4) compatible with an acute motor and sensory axonal neuropathy (AMSAN), and only partial clinical improvement after IVIG administration ( $2 \mathrm{~g} / \mathrm{kg}$ for 5 days). This case was previously reported [5] but EDX was not available at that time. In the three other cases, the EDX (S1-S3) was consistent with acute inflammatory demyelinating polyneuropathy (AIDP) and no serum anti-ganglioside antibodies were detected. Two of the other patients (case1 and case2) presented severe SARS-CoV-2 infection and had good clinical outcome after
IVIG treatment ( $2 \mathrm{~g} / \mathrm{kg}$ for 5 days). The third other patient (case3) had mild SARS-CoV-2 infection and had spontaneously favorable evolution without treatment.

\section{Discussion}

We describe four cases of SARS-CoV-2-associated GBS, adding evidence to the probable association between these two entities. The lag time between COVID-19 infection and the neurological onset, the response to IVIG, and particularly the presence of anti-ganglioside antibodies in one patient are highly suggestive of a post-infectious immunemediated mechanism.

Our patient with positive anti-ganglioside antibodies (anti-GD1b IgG) was the only one with brainstem involvement, suggestive of GBS/Bickerstaff brainstem encephalitis (BBE) overlap, and with electrophysiological features suggestive of AMSAN, a previously reported association [6]. The three other patients presented with AIDP and had negative anti-ganglioside screening. While our patient presented myoclonus limited to the palatal region, myoclonus has already been described in BBE [7]. Anti-GD1b have been described in the clinical spectrum of GBS-classically in acute sensory ataxic neuropathy (ASAN), but also in AMSAN-, MFS and BBE, and are associated with more severe disease and slower recovery [5, 8, 9]. Although, the 7 -week delay of EDX raises the question of a probable critical illness polyneuropathy component-which is difficult to distinguish from an AMSAN- the other features and the response to IVIG are in favor of a post-infectious immunemediated process.

According to a recent review of the literature, anti-ganglioside antibodies in COVID-19-associated GBS were positive in only 5/36 patients (14\%) [3]. Four new anti-ganglioside positive cases, including one of our patients, were identified reviewing the literature for this paper and are summarized in Table 2. In COVID-19 patients, anti-ganglioside antibodies were found not only in patients with GBS but also in patients with variable neurological presentations (cranial neuropathy with meningo-polyradiculitis, choreic movements, myelitis) $[5,8-10]$ which casts doubt on whether these are truly pathogenic in all cases. Interestingly, anti-ganglioside antibodies have been described in other neurological diseases besides the GBS/MFS/BBE spectrum, even though their clinical significance remains unsure [11]. On the other hand, the role of anti-ganglioside antibodies in the genesis of axonal subtypes of GBS after Campylobacter jejuni infection is well established, and associated with severe disability [12].

Prevalence of anti-ganglioside antibodies in GBS was, respectively, $31.7 \%$ and $50 \%$ in two large cohorts of, respectively, 306 and 119 patients [13, 14]. The current low rate of positive anti-ganglioside antibodies in 


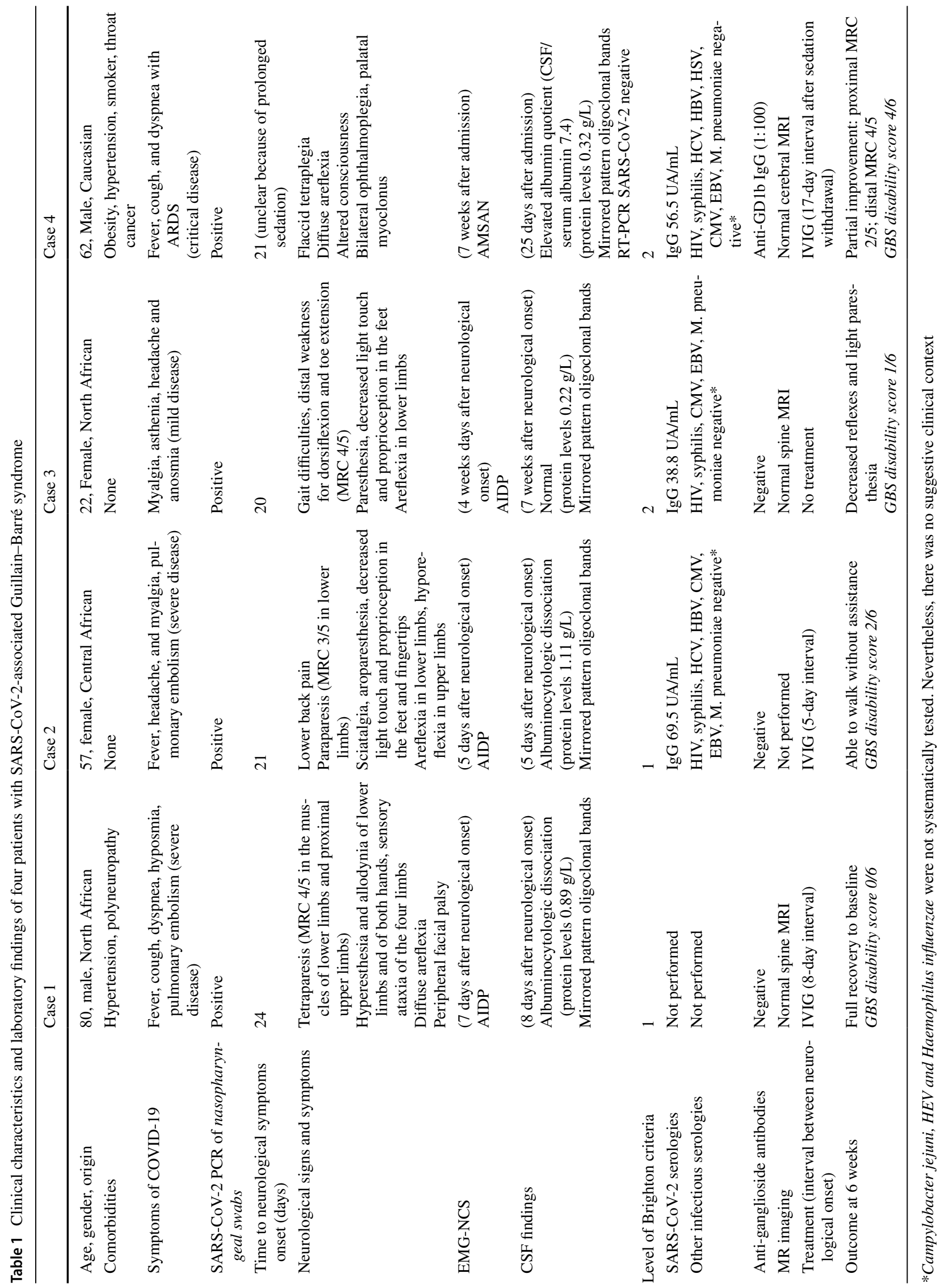


Table 2 Anti-ganglioside antibodies in SARS-CoV2-associated neurological syndromes

\begin{tabular}{lll}
\hline Authors & Neurological syndromes & Anti-ganglioside antibodies (titer) \\
\hline Gutierrez [9] & MFS & GD1b (NA) \\
Lantos [10] & MFS & Asialo GM1 (“equivocal range”) \\
Gigli [18] & GBS (AIDP) & GD1a (NA) \\
Chan [19] & GBS (EDX deferred) & GM2 (NA) \\
Dufour [3] & GBS (EDX deferred) & Asialo GM1 (1:76), GM1 (1:58), \\
& & GD1A (1:76), GD1b (1:60), GQ1b \\
& & (1:56) \\
Tatu [20] & GBS (AIDP) & GM1 (NA), GM2 (NA) \\
Masuccio [8] & GBS (AMAN) and myelitis & GD1b (NA) \\
Kopscik [21] & MFS & GQ1b (1:100) \\
Civardi [22] & GBS (AIDP) & GM1 (1:70), GD1a (1:72), GD1b (1:64) \\
Guilmot [5] & GBS (AMSAN) with brainstem involvement & GD1b (> 1:100) \\
Guilmot [5] & Cranial neuropathy with meningo-polyradiculitis & GD1b (>1:100) \\
Guilmot [5] & Choreic movements & GD1b (>1:100) \\
\hline
\end{tabular}

SARS-CoV-2-associated GBS could be underestimated due to lack of testing, or due to the fact that a majority of reported SARS-CoV-2-associated GBS are of the AIDP variant (48/62 [77.4\%];40/75 [75\%]) [1, 15], which is known to be less frequently associated with serum anti-ganglioside antibodies than the axonal forms [12, 13, 15-17]. Another explanation might be the presence of antibodies against untested gangliosides, ganglioside complexes or other more atypical antigens. Anti-ganglioside complex antibodies are difficult to detect and are not tested in routine clinical practice but can be useful in patient with negative antibodies against single gangliosides [18]. It may be hypothesized that the SARS-CoV-2 spike-bearing gangliosides could form a complexed neoantigen recognized by unidentified specific anti-complex antibodies. On the other hand, antibodies less typically associated with GBS could also have a pathophysiological role. Positive anti-CASPR2 antibodies have been reported in rare cases of GBS [9], and one patient suffering from an encephalitis following SARS-CoV-2 infection had positive anti-CASPR2 antibodies [5]. Currently, antiCASPR2 antibodies are not routinely screened in GBS patients and might thus be underreported.

Finally, other mechanisms such as the 'cytokine storm' could hypothetically be involved in the pathogenesis of SARS-CoV-2-associated GBS, especially in the acute onset within days after onset of viral infection, since many of the released cytokines have been implicated in the pathogenesis of 'classical' GBS [19].

\section{Conclusion}

We report four cases of SARS-CoV-2-associated GBS. The interval of 3 weeks between SARS-CoV-2 symptoms and neurological onset, the favorable response to IVIG, and the positive anti-ganglioside in one patient further support the hypothesis of an immune-mediated post-infectious process. Further autoantibody testing (including anti-ganglioside, anti-neuronal antibodies like anti-CASPR2, or anti-complex antibodies) in SARS-CoV-2 patients exhibiting neurological symptoms could allow a better understanding of these probable immune-mediated post-infectious processes.

Supplementary Information The online version contains supplementary material available at https://doi.org/10.1007/s13760-021-01787-y.

Acknowledgements The authors wish to thank the patients for providing permission to share their information.

Author contributions Study concept and design: AG, MG. Acquisition and interpretation of data: all the authors. Drafting of the manuscript: AG. Critical revision of the manuscript for important intellectual content: all the authors. All the authors read and approved the final manuscript.

Funding No targeted funding reported.

Data availability MG had full access to all the data in the study and takes responsibility for the integrity of the data and the accuracy of the data analysis.

\section{Declarations}

Conflict of interest The authors declare that they have no conflicts of interest.

Ethical approval All the procedures were performed in accordance with the institutional ethics committee and the Declaration of Helsinki. 


\section{References}

1. Abu-Rumeileh S, Abdelhak A, Foschi M et al (2020) GuillainBarré syndrome spectrum associated with COVID-19: an up-todate systematic review of 73 cases. J Neurol. https://doi.org/10. 1007/s00415-020-10124-x

2. Keddie S, Pakpoor J, Mousele C et al (2020) Epidemiological and cohort study finds no association between COVID-19 and Guillain-Barré syndrome. Brain. https://doi.org/10.1093/brain/ awaa433

3. Dufour C, Co T-K, Liu A (2021) GM1 ganglioside antibody and COVID-19 related Guillain Barre Syndrome - A case report, systemic review and implication for vaccine development. Brain Behav Immun Health 12:100203. https://doi.org/10.1016/j.bbih. 2021.100203

4. Dalakas MC (2020) Guillain-Barré syndrome: The first documented COVID-19-triggered autoimmune neurologic disease: More to come with myositis in the offing. Neurol Neuroimmunol Neuroinflamm 7:e781. https://doi.org/10.1212/NXI.0000000000 000781

5. Guilmot A, Maldonado Slootjes S, Sellimi A et al (2020) Immunemediated neurological syndromes in SARS-CoV-2-infected patients. J Neurol. https://doi.org/10.1007/s00415-020-10108-x

6. Pegg EJ, Chhetri SK, Lekwuwa UG, Majeed T (2016) An overlapping case of miller fisher syndrome, bickerstaff's encephalitis, and the ASMAN variant of guillain-barre syndrome. Case Rep Neurol Med 2016:1596850. https://doi.org/10.1155/2016/1596850

7. Takaki R, Nagasaka T, Suwa Y et al (2017) A case of Bickerstaff brainstem encephalitis with transient reflex myoclonus. Rinsho Shinkeigaku 57:395-398. https://doi.org/10.5692/clinicalneurol. cn-001026

8. Masuccio FG, Barra M, Claudio G, Claudio S (2020) A rare case of acute motor axonal neuropathy and myelitis related to SARS-CoV-2 infection. J Neurol. https://doi.org/10.1007/ s00415-020-10219-5

9. Gutiérrez-Ortiz C, Méndez A, Rodrigo-Rey S et al (2020) Miller Fisher Syndrome and polyneuritis cranialis in COVID-19. Neurology. https://doi.org/10.1212/WNL.0000000000009619.10.1212/ WNL.0000000000009619

10. Lantos JE, Strauss SB, Lin E (2020) COVID-19-Associated Miller Fisher Syndrome: MRI Findings. AJNR Am J Neuroradiol 41:1184-1186. https://doi.org/10.3174/ajnr.A6609

11. Wanleenuwat P, Iwanowski P, Kozubski W (2020) Antiganglioside antibodies in neurological diseases. J Neurol Sci 408:116576. https://doi.org/10.1016/j.jns.2019.116576

12. Islam Z, Jacobs BC, van Belkum A et al (2010) Axonal variant of Guillain-Barre syndrome associated with Campylobacter infection in Bangladesh. Neurology 74:581-587. https://doi.org/10.1212/ WNL.0b013e3181cff735

13. Caudie C, Quittard Pinon A, Taravel D et al (2011) Preceding infections and anti-ganglioside antibody profiles assessed by a dot immunoassay in 306 French Guillain-Barré syndrome patients. J Neurol 258:1958-1964. https://doi.org/10.1007/ s00415-011-6042-9

14. Kim JK, Bae JS, Kim D-S et al (2014) Prevalence of anti-ganglioside antibodies and their clinical correlates with Guillain-Barré syndrome in Korea: a nationwide multicenter study. J Clin Neurol 10:94-100. https://doi.org/10.3988/jcn.2014.10.2.94

15. Hasan I, Saif-Ur-Rahman KM, Hayat S et al (2020) Guillain-Barré syndrome associated with SARS-CoV-2 infection: A systematic review and individual participant data meta-analysis. J Peripher Nerv Syst 25:335-343. https://doi.org/10.1111/jns.12419

16. Van den Bergh PYK, Piéret F, Woodard JL et al (2018) GuillainBarrÉ syndrome subtype diagnosis: A prospective multicentric European study. Muscle Nerve. https://doi.org/10.1002/mus. 26056

17. Sedano MJ, Orizaola P, Gallardo E et al (2019) A unicenter, prospective study of Guillain-Barré syndrome in Spain. Acta Neurol Scand 139:546-554. https://doi.org/10.1111/ane.13092

18. Goodfellow JA, Willison HJ (2016) Antiganglioside, antiganglioside-complex, and antiglycolipid-complex antibodies in immunemediated neuropathies. Curr Opin Neurol 29:572-580. https://doi. org/10.1097/WCO.0000000000000361

19. Hussain FS, Eldeeb MA, Blackmore D, Siddiqi ZA (2020) Guillain Barré syndrome and COVID-19: possible role of the cytokine storm. Autoimmun Rev 19:102681. https://doi.org/10.1016/j. autrev.2020.102681

20. Tatu L, Nono S, Grácio S, Koçer S (2021) Guillain-Barré syndrome in the COVID-19 era: another occasional cluster? J Neurol 268(4):1198-1200. https://doi.org/10.1007/s00415-020-10005-3

21. Kopscik M, Giourgas B, Presley B (2020) A case report of acute motor and sensory polyneuropathy as the presenting symptom of SARS-CoV-2. Clin Pract Cases Emerg Med 4(3):352-354. https:// doi.org/10.5811/cpcem.2020.6.48683

22. Alessandra CC, Dinha C, Geda J, Geda C (2020) Antiganglioside antibodies in Guillain-Barré syndrome associated with SARSCoV-2 infection. J Neurol Neurosurg Psychiatry 91(12):13611362. https://doi.org/10.1136/jnnp-2020-324279

Publisher's Note Springer Nature remains neutral with regard to jurisdictional claims in published maps and institutional affiliations. 